\title{
Soya protein stimulates bile acid excretion by the liver and intestine through direct and indirect pathways influenced by the presence of dietary cholesterol
}

\author{
Gloria Leticia Arellano-Martínez ${ }^{1,2}$, Omar Granados ${ }^{1}$, Berenice Palacios-González ${ }^{1}$, \\ Nimbe Torres ${ }^{1}$, Isabel Medina-Vera ${ }^{1}$ and Armando R. Tovar ${ }^{1 *}$ \\ ${ }^{1}$ Departamento de Fisiología de la Nutrición, Instituto Nacional de Ciencias Médicas y Nutrición Salvador Zubirán, \\ Mexico City, DF 14000, Mexico \\ ${ }^{2}$ Doctorado en Ciencias Bioquímicas, Facultad de Quimica, Universidad Nacional Autónoma de México, México City, \\ DF 04510, Mexico \\ (Submitted 17 June 2013 - Final revision received 15 January 2014 - Accepted 23 January 2014 - First published online 25 March 2014)
}

\section{Abstract}

Several studies using different animal models have demonstrated that the consumption of soya protein (SP) reduces serum cholesterol concentrations by increasing the excretion of bile acids (BA). However, the mechanism by which SP enhances BA excretion is not fully understood. Therefore, the aim of the present study was to determine whether the consumption of SP regulates the expression of key enzymes involved in hepatic BA synthesis and the transporters involved in reverse cholesterol transport (RCT) via fibroblast growth factor 15 (FGF15) and/or small heterodimer protein (SHP) in rats. To achieve this aim, four groups of rats were fed experimental diets containing $20 \%$ casein (C) or SP with or without the addition of $0 \cdot 2 \%$ cholesterol and the expression of hepatic genes involved in BA synthesis and the ileal and hepatic RCT was measured. Rats fed the SP diet had higher concentrations of ileal FGF15 and hepatic FGF15 receptor (FGFR4) and increased expression of SHP and liver receptor homolog 1 (LRH1) than those fed the C diet; as a result, the excretion of faecal BA was greater. The addition of cholesterol to the diet repressed the protein abundance of FGF15 and FGFR4; however, SP increased the expression of SHP and LRH1 to a lesser extent. Nonetheless, the expression of $A B C G 5 / 8$ was increased in the intestine of rats fed the SP diet, and the effect was enhanced by the addition of cholesterol to the diet. In conclusion, SP in the presence of cholesterol increases BA synthesis via the repressions of FGF15 and SHP and accelerates BA excretion to prevent cholesterol overload in the enterocytes by increasing RCT.

\section{Key words: Bile acids: Cholesterol: Fibroblast growth factor 15: Fibroblast growth factor 15 receptor 4: Intestine: Rats:} Soya protein

Several meta-analyses have demonstrated that the consumption of soya protein (SP) reduces the concentration of total cholesterol, especially that of LDL-cholesterol, in humans. In some instances, it increases the concentration of HDL-cholesterol ${ }^{(1-3)}$. Several mechanisms have been proposed to explain how SP can reduce the concentration of cholesterol $^{(4,5)}$. Among other possible mechanisms, the data obtained from animal studies, mainly using rats and rabbits, suggest that SP increases the excretion of bile acids (BA), lowering the serum concentration of cholesterol ${ }^{(5-7)}$.

BA are produced via the neutral (classic) pathway or the acidic (alternative) pathway ${ }^{(8)}$. The neutral pathway is controlled by the rate-limiting enzyme $7 \alpha$-hydroxylase (also called CYP7A1) ${ }^{(9)}$, whereas the flux through the alternative pathway is regulated by the enzyme $27 \alpha$-hydroxylase $(\mathrm{CYP} 27)^{(10)}$. The expression of $C Y P 7 A 1$ is regulated by a member of the transcription factor family: liver $\mathrm{X}$ receptors or LXR $\alpha^{(11)}$. LXR are nuclear hormone receptors that form active heterodimers with retinoid $\mathrm{X}$ receptors, which are activated by oxysterols and serve as key sensors of intracellular sterol levels by regulating the expression of the genes that control cholesterol absorption, storage, transport and elimination. It is known that $\operatorname{LXR} \alpha$ up-regulates the expression of CYP7A1 by increasing the production of $\mathrm{BA}^{(11)}$.

In mice, the expression of $C Y P 7 A 1$ is down-regulated by the nuclear receptor farnesoid $\mathrm{X}$ receptor (FXR), which is activated by the presence of its natural ligands, $\mathrm{BA}^{(12)}$. Thus, when there is an increase in the enterohepatic circulation of

Abbreviations: ABCG5, ATP-binding cassette, subfamily G, member 5; ABCG8, ATP-binding cassette, subfamily G, member 8; BA, bile acids; C, 20\% casein; CC, $20 \%$ casein plus $0 \cdot 2 \%$ cholesterol; CYP7A1, $7 \alpha$-hydroxylase; CYP27, $27 \alpha$-hydroxylase; FGF15, fibroblast growth factor 15 ; FGFR4, fibroblast growth factor receptor 4; FXR, farnesoid X receptor; LRH1, liver receptor homolog 1; LXR, liver X receptors; RCT, reverse cholesterol transport; S, $20 \%$ soya protein; SC, $20 \%$ soya protein plus $0.2 \%$ cholesterol; SHP, small heterodimer protein; SP, soya protein; SREBP1c, sterol regulatory element-binding protein-1c. 
BA, these acids activate FXR, which in turn stimulates the transcription of another nuclear receptor, small heterodimer protein (SHP). The expression of $S H P$ represses the transcription of CYP7A1 by competing with the nuclear receptor liver receptor homolog 1 (LRH1), which is a constitutive activator of the CYP7A1 gene ${ }^{(13,14)}$. However, it has been demonstrated that CYP27 activity is insensitive to the size of the hepatic BA pool $^{(15)}$. More recent studies have established that the expression of $C Y P 7 A 1$ in mice is also regulated by fibroblast growth factor 15 (FGF15), which is the orthologue of the human FGF19 (fibroblast growth factor 19) and is synthesised in the intestine. The expression of FGF15 is stimulated in the small intestine by the nuclear BA receptor FXR and it is released into the circulation. It is then recognised by a specific receptor in the liver, fibroblast growth factor receptor 4 (FGFR4), leading to an increase in the expression of $S H P^{(16)}$. As a consequence, there is a reduction in the expression of $C Y P 7 A 1$ and therefore in the synthesis of BA.

On the other hand, the synthesis of FGF15 in the intestine, particularly in the ileal portion, is controlled by the transcription factor FXR. This nuclear receptor also regulates the expression of several important proteins involved in the transport and mobilisation of BA in the enterocytes. These proteins, the ileal BA transporter and the ileal BA-binding protein play key roles in the uptake and mobilisation of BA from the enterocytes into the circulation to transport them into the liver via enterohepatic circulation ${ }^{(17)}$. In addition, the concentration of $\mathrm{BA}$, as well as that of cholesterol in the enterocytes, is also regulated by the specific transporters that belong to the ATP cassette-binding transporter family ABCG5/8 (ATP-binding cassette, subfamily G, member 5/8) ${ }^{(18)}$.

However, there is scarce evidence as to whether SP regulates the synthesis of BA by inducing metabolic adaptations in the liver and intestine. Therefore, the aim of the present study was to determine whether the consumption of SP increases faecal BA excretion by increasing BA synthesis via SHP and/or FGF15.

\section{Experimental methods}

\section{Animals and diets}

The animal protocol described below was approved by the Animal Committee of the National Institute of Medical Sciences and Nutrition, Mexico City. A total of forty-eight male Wistar rats (Harlan-Teklad) weighing $170 \mathrm{~g}$ each were randomly divided into four groups. The rats were housed individually in stainless-steel cages and placed in temperature- and humiditycontrolled rooms under a $12 \mathrm{~h}$ light $-12 \mathrm{~h}$ dark cycle. The rats had free access to water throughout the study. The rats in each group were fed ad libitum for $3 \mathrm{~d}$ to acclimatise them to one of the following diets: (1) $20 \%$ casein (C) (Harlan-Teklad Research Diets); (2) $20 \%$ soya protein (S) (Supro 710; Solae); (3) $20 \%$ casein plus $0 \cdot 2 \%$ cholesterol (CC); (4) $20 \%$ soya protein plus $0.2 \%$ cholesterol (SC). The composition of isoflavones in the SP was as follows ( $\mathrm{mg} / \mathrm{g}$ of protein): genistein 1.38; daidzein $0 \cdot 71$; glycitein $0 \cdot 19$. On the 4 th day of the study, each group was subjected to time-restricted feeding (food was available beginning at 09.00 hours and removed at 14.00 hours) with one of the four experimental diets for 1 week. The rats were killed after an overnight fast. Body weight and food intake were recorded daily. The rats were killed by decapitation after $\mathrm{CO}_{2}$ anaesthetisation. Blood was collected in tubes with a gel and clot activator (BD) and centrifuged at $1000 \mathrm{~g}$ for $10 \mathrm{~min}$; serum was separated and stored at $-70^{\circ} \mathrm{C}$. The liver was removed, weighed and immediately frozen and stored at $-80^{\circ} \mathrm{C}$ until the extraction of total RNA or total protein. The ileum was quickly removed and immediately frozen and stored at $-80^{\circ} \mathrm{C}$ until the extraction of total RNA or total protein. Faecal samples were collected for $3 \mathrm{~d}$ before the end of the experimental period.

\section{Determination of cholesterol and TAG concentrations}

Hepatic and faecal lipids were extracted with chloroform-methanol using the Folch method ${ }^{(19)}$. Total serum, faecal, and hepatic cholesterol and TAG concentrations were measured using a colorimetric kit. The colorimetric kit for TAG and cholesterol assays was obtained from DiaSys Diagnostic Systems GmbH.

\section{Determination of bile acid concentrations}

Total BA from freeze-dried faecal samples were extracted with $t$-butanol and measured enzymatically with $3 \alpha$-hydroxysteroid dehydrogenase (Sigma) according to the method of Turley \& Dietschy ${ }^{(20)}$.

\section{Real-time $P C R$}

Total RNA was extracted from the liver and ileum samples according to the method described by Chomczynski \& Sacchi ${ }^{(21)}$ and pooled. To carry out an integrity analysis, $15 \mu \mathrm{g}$ of total RNA were separated using $1.0 \%$ agarose $/ 2 \cdot 2 \mathrm{M}$-formaldehyde gel electrophoresis. Samples of $300 \mathrm{ng}$ of total RNA were subjected to reverse transcription and PCR amplification using Master Mix (Applied Biosystems). Parallel non-template control reactions were run in the absence of RNA to assess the absence of nucleic acid contamination in the reaction mixtures. TaqMan fluorogenic probes and oligonucleotide primers were obtained from Applied Biosystems (SHP (Rn00589173_ml), LRH1 (Rn00572649_m1), LXR (Rn00581185_m1), sterol regulatory element-binding protein-1c (SREBP1c) (Rn 01495769_m1), CYP7A1 (Rn00564065_m1), CYP27 (Rn00710297_m1), ABCG5 (Rn), ABCG8 (Rn00590367_m1), ATP-binding cassette transporter A1 (ABCA1) (Rn00710172_m1) and ileal bile acid protein (IBAP) (Rn00567314_m1)). TaqMan PCR assays for each target gene were carried out in triplicate in ninety-six-well optical plates with the ABI Prism 7000 Sequence Detection System (Perkin-Elmer Applied Biosystems) following the manufacturer's protocol. The relative amounts of all mRNA were calculated using the comparative CT method (User Bulletin no. 2; PE Applied Biosystems). For the liver and intestine analyses, $\beta$-actin was used as the invariant control.

\section{Western blot analysis}

Extracts of the rat liver and ileum were prepared by homogenising the tissue in a lysis buffer with $50 \mathrm{~mm}$-Tris, $\mathrm{pH} 7 \cdot 4$, 
0.27 M-sucrose, $1 \mathrm{~mm}$-sodium orthovanadate, $\mathrm{pH} 10,1 \mathrm{~mm}$ EDTA, $1 \mathrm{~mm}$-ethylene glycol tetraacetic acid, $10 \mathrm{~mm}$-sodium $\beta$-glycerophosphate, $50 \mathrm{~mm}-\mathrm{NaF}, 5 \mathrm{~mm}$-sodium pyrophosphate, $1 \%(\mathrm{w} / \mathrm{v})$ Triton $\mathrm{X}-100,0 \cdot 1 \%(\mathrm{v} / \mathrm{v})$ 2-mercaptoethanol and protease inhibitor cocktail tablets. The samples were stored at $-20^{\circ} \mathrm{C}$ until analysis. The proteins $(40 \mu \mathrm{g})$ were separated electrophoretically on a $7 \%$ SDS-polyacrylamide gel and transferred onto polyvinylidene difluoride membranes. The membranes were incubated with specific antibodies against total FGFR4 or total FGF15 and detected with an anti-rabbit secondary antibody. A $\beta$-actin antibody was used as a loading control. The labelled bands were detected using chemiluminescence with the visualiser Spray \& Glow Western blotting detection system. The chemiluminescence was quantified using optical densitometry and analysed with the Image $144 \mathrm{p}$ digital image processing software (National Institutes of Health).

\section{Statistical analysis}

Values are expressed as means with their standard errors. All the parameters were evaluated with a two-way ANOVA using the type of protein and the cholesterol content in the diet as independent variables, followed by Fisher's protected least significant difference test. Differences were considered to be statistically significant at $P<0.05$ and are indicated by letters in the figures $(\mathrm{a}>\mathrm{b}>\mathrm{c})$.

\section{Results}

\section{Weight gain and food intake}

At the beginning of the study, rats from the four experimental groups were not significantly different. However, at the end of the study, rats fed the SC diet tended to gain less weight, although the difference was not significant $(P=0.052)$. In addition, the average food and energy intake was similar in all the four groups, but as expected, cholesterol intake was higher in the groups that consumed diets containing cholesterol (Table 1).

\section{Serum and hepatic lipid concentrations}

Rats fed the CC diet had significantly higher serum cholesterol concentrations than those in the other groups (Table 1). The two-way ANOVA indicated that the type of protein consumed and the cholesterol content in the diet had a significant effect on serum cholesterol concentrations. Rats fed the $S$ or SC diet had a serum cholesterol concentration that was approximately $15 \%$ lower than that of the corresponding groups fed the $\mathrm{C}$ or $\mathrm{CC}$ diet. Serum TAG content followed the opposite pattern: rats fed the $\mathrm{C}$ and $\mathrm{S}$ diets had significantly higher concentrations than those fed the CC and SC diets, indicating that the cholesterol content in the diet rather than the type of protein consumed reduced serum TAG concentrations (Table 1).

Interestingly, the liver weight of rats fed the S or SC diet was approximately $10 \%$ lower than that of rats fed the C or CC. As expected, rats fed diets with $0 \cdot 2 \%$ cholesterol had significantly higher hepatic cholesterol concentrations than those fed diets without cholesterol. However, rats fed the SC diet had $42 \%$ lower hepatic cholesterol concentrations than those fed the CC diet $(P<0 \cdot 05)$. Rats fed the SC diet accumulated approximately 6.4 times more cholesterol in their livers than those fed the $\mathrm{S}$ diet, and rats fed the CC diet accumulated $7 \cdot 4$ times more cholesterol than those fed the $\mathrm{C}$ diet. There was no significant difference between rats fed the $\mathrm{C}$ diet and those fed the $\mathrm{S}$ diet. Hepatic TAG concentrations followed a similar pattern: rats fed the SC diet had 35\% lower TAG concentrations than those fed the CC diet, and TAG concentrations in the liver of rats fed the CC or SC diet were $4 \cdot 1$ and 3.5 times lower than those in rats fed the $\mathrm{C}$ and $\mathrm{S}$ diets, respectively (Table 1 ).

Hepatic gene expression of SHP, liver receptor homolog 1, liver $X$ receptors, sterol regulatory element-binding protein-1c, $7 \alpha$-hydroxylase and $27 \alpha$-hydroxylase

To explore BA synthesis, we determined the relative expression of SHP, LRH1, LXR, SREBP1C, CYP7A1 and CYP27 mRNA in the liver of rats in the four groups. The results

Table 1. Body and liver weights and serum and hepatic lipid concentrations in rats fed casein or soya protein diets with or without $0.2 \%$ cholesterol (Chol) (Mean values with their standard errors; $n$ )

\begin{tabular}{|c|c|c|c|c|c|c|c|c|c|c|c|}
\hline & \multicolumn{2}{|c|}{ C } & \multicolumn{2}{|c|}{$S$} & \multicolumn{2}{|c|}{$\mathrm{CC}$} & \multicolumn{2}{|c|}{ SC } & \multicolumn{3}{|c|}{$P$} \\
\hline Initial weight $(\mathrm{g})$ & $168 \cdot 36$ & $5 \cdot 79$ & $168 \cdot 11$ & 8.35 & $170 \cdot 42$ & $5 \cdot 64$ & $168 \cdot 85$ & $6 \cdot 52$ & NS & NS & NS \\
\hline Average food intake $(\mathrm{g})$ & $12 \cdot 49$ & 0.6 & $11 \cdot 77$ & 0.7 & $12 \cdot 77$ & 0.66 & $11 \cdot 3$ & 0.65 & NS & NS & NS \\
\hline Average energy intake (kJ) & 209.4 & 10 & $197 \cdot 3$ & 12 & $216 \cdot 5$ & 11.3 & 191.4 & 11.3 & NS & NS & NS \\
\hline Average Chol intake $(\mathrm{g})$ & 0.00 & 0.00 & 0.00 & 0.00 & 0.064 & 0.004 & 0.057 & 0.004 & NS & 0.0001 & NS \\
\hline TAG (mM) & $2 \cdot 38^{a}$ & 0.039 & $1.75^{b}$ & 0.045 & $0.68^{\mathrm{C}}$ & 0.012 & $0.70^{c}$ & 0.027 & $<0.0001$ & $<0.0001$ & $<0.0001$ \\
\hline \multicolumn{12}{|l|}{ Liver } \\
\hline Weight (g) & $7 \cdot 6$ & 0.45 & $6 \cdot 4$ & 0.36 & $7 \cdot 7$ & 0.53 & $6 \cdot 0$ & 0.24 & & & \\
\hline $\mathrm{Chol}(\mathrm{mmol} / \mathrm{g})$ & $11.56^{\mathrm{c}}$ & 2.07 & $7 \cdot 73^{c}$ & 0.78 & $85 \cdot 35^{\mathrm{a}}$ & $12 \cdot 41$ & $49 \cdot 68^{b}$ & 5.95 & 0.0073 & $<0.0001$ & 0.0283 \\
\hline TAG $(\mathrm{mmol} / \mathrm{g})$ & $23.04^{\mathrm{c}}$ & $4 \cdot 74$ & $17 \cdot 73^{\mathrm{c}}$ & $2 \cdot 48$ & $94.49^{a}$ & $13 \cdot 21$ & $61.53^{b}$ & 5.42 & 0.02 & $<0.0001$ & 0.08 \\
\hline
\end{tabular}

C, $20 \%$ casein; S, $20 \%$ soya protein; CC, $20 \%$ casein plus $0.2 \%$ Chol; SC, $20 \%$ soya protein plus $0.2 \%$ Chol.

$\mathrm{a}, \mathrm{b}, \mathrm{c}, \mathrm{d}$ Mean values within a row with unlike superscript letters were significantly different $(P<0.05 ; \mathrm{a}>\mathrm{b}>\mathrm{c}>\mathrm{d})$. 
revealed that the expression of SHP and LRH1 mRNA was higher in rats fed the SP diet regardless of the cholesterol content in the diet. As expected, the presence of cholesterol in the diet reduced the expression of $S H P$ and $L R H 1$ (Fig. 1(A) and (B)). The expression of $C Y P 7 A 1$ mRNA was significantly higher in the liver of rats fed diets with cholesterol than in that of rats fed diets without cholesterol (Fig. 1(C)). Interestingly, rats fed the SC diet had significantly higher expression of CYP7A1 than those fed the CC diet (Fig. 1(C)). There were no significant differences in the expression of CYP27 among the groups (Fig. 1(D)). On the other hand, the expression of $L X R$ and SREBP $1 C$ mRNA was significantly lower in the liver of rats fed the SP diet, regardless of the cholesterol content in the diet (Fig. 1(E) and (F)).

\section{Concentrations of ileal fibroblast growth factor 15, hepatic fibroblast growth factor receptor 4 and faecal bile acid}

To determine whether SP consumption has an independent mechanism of SHP action, we measured the concentrations of FGF15 and FGFR4 in the ileum and liver, respectively. The results indicated that the consumption of SP increased FGF15 concentrations, but FGF15 was not detected in rats fed diets without cholesterol (Fig. 2(A)). Interestingly, rats fed the CC diet had FGFR4 concentrations that were $0 \cdot 22$ times lower than those of rats fed the $\mathrm{C}$ diet, and rats fed the SC diet had FGFR4 concentrations that were 1.0 times lower than those of rats fed the $\mathrm{S}$ diet (Fig. 2(B)).

Total faecal BA excretion was measured in the faecal samples collected for $3 \mathrm{~d}$ before the end of the experimental period. As shown in Fig. 2(C), rats fed the SC diet excreted significantly more BA during the study period than those fed the CC diet. At the end of the study, rats fed the SC diet excreted 0.94 times more BA than those fed the CC diet $(P<0.05)$. Interestingly, rats fed the $\mathrm{S}$ diet also excreted 1.5 times more BA than those fed the $\mathrm{C}$ diet. The two-way ANOVA revealed that the type of protein consumed and the cholesterol content in the diet had a significant effect on faecal BA excretion. In addition, the interaction between the type of protein consumed and the cholesterol content in the diet was highly significant.

\section{Hepatic gene expression of ATP-binding cassette transporter A1 and ATP-binding cassette, subfamily G, member 5 and serum HDL and LDL concentrations}

To evaluate reverse cholesterol transport (RCT), we measured the expression of $A B C A 1$ and $A B G C 5$ in the liver.
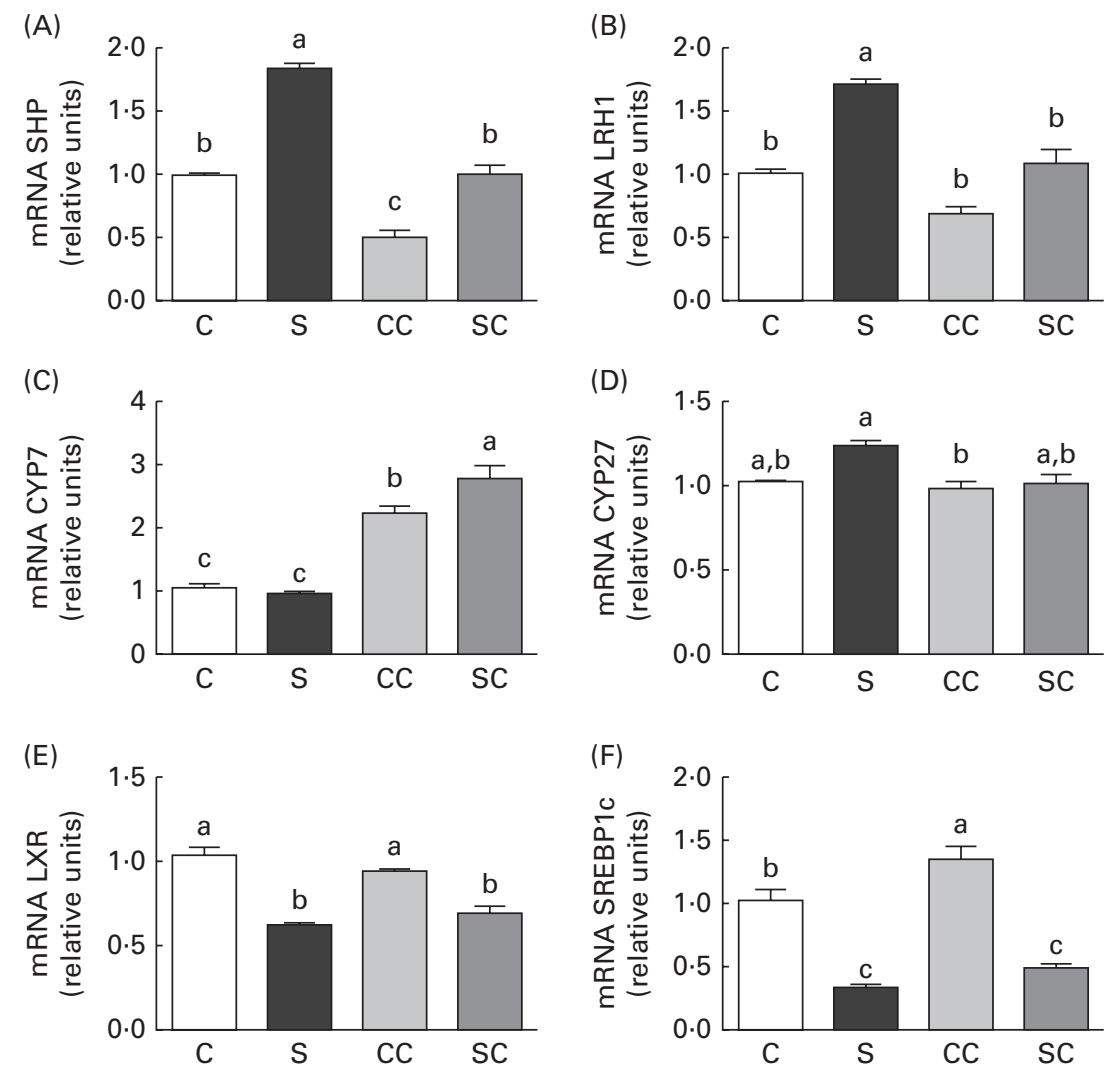

Fig. 1. Effects of the consumption of a soya protein diet with $0.2 \%$ cholesterol (SC) and without $0.2 \%$ cholesterol (S) for $9 \mathrm{~d}$ on the expression of $(\mathrm{A}) \mathrm{SHP}$, (B) liver receptor homolog 1 (LRH1), (C) $7 \alpha$-hydroxylase (CYP7A1), (D) 27 $\alpha$-hydroxylase (CYP27), (E) liver X receptor (LXR) and (F) sterol regulatory elementbinding protein-1c (SREBP1C) in the liver of rats ( $n 12$ rats per group). Values are means, with their standard errors represented by vertical bars. ${ }^{\text {,b,c }}$ Mean values with unlike letters were significantly different $(P<0.05)$. C, $20 \%$ casein; CC, $20 \%$ casein plus $0.2 \%$ cholesterol. 
(A)
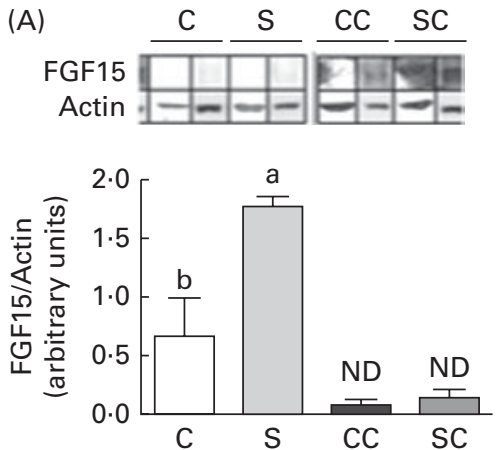

(B)

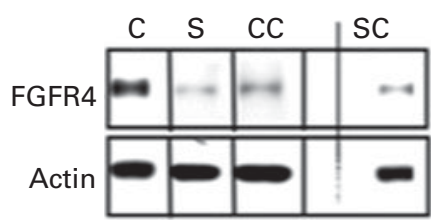

Fig. 2. Effects of the consumption of a soya protein diet with $0.2 \%$ cholesterol (SC) and without $0.2 \%$ cholesterol (S) for $9 \mathrm{~d}$ on (A) fibroblast growth factor 15 (FGF15) concentrations in the ileum, (B) fibroblast growth factor receptor 4 (FGFR4) concentrations in the liver and (C) total bile acid concentrations in the faeces ( $n 12$ rats per group). $P$ values for protein $(<0.0001)$, cholesterol $(<0.0001)$ and protien $\times$ cholesterol $(0.0057)$. Values are means, with their standard errors represented by vertical bars. ${ }^{\text {a,b,c,d }}$ Mean values with unlike letters were significantly different $(P<0.05)$. C, $20 \%$ casein; CC, $20 \%$ casein plus $0.2 \%$ cholesterol; ND, not detected.

The expression of $A B C A 1$ was significantly increased only in rats fed diets containing $0.2 \%$ cholesterol (Fig. 3(A)). There were no significant differences in the abundance of $A B C G 5$ mRNA among the four groups (Fig. 3(B)).

To determine whether the consumption of SP regulates HDL and/or LDL concentrations, we measured the concentrations. The results indicated that the consumption of SP does not alter HDL concentrations (Fig. 3(C)). However, LDL concentrations in rats fed the SC diet were $2 \cdot 0$ times lower than those in rats fed the CC diet (Fig. 3(D)).
Intestinal gene expression of ATP-binding cassette, subfamily G, member 5, ATP-binding cassette, subfamily $G$, member 8, and ileal bile acid-binding protein and total faecal cholesterol concentrations

We studied the effect of SP on the expression of several genes in the ileal portion of the intestine. The expression of the $A B C G 5$ and $A B C G 8$ transporters was significantly stimulated by the cholesterol and SP content in the diet, and the effect was additive, indicating that the up-regulation of the expression of these transporter genes by SP was enhanced when rats consumed diets containing cholesterol (Fig. 4(A) and (B)). There were no significant differences in the expression of the ileal BA-binding protein $(I B A B P)$ among the groups (Fig. 4(C)). Interestingly, rats fed the SP diet had significantly higher faecal cholesterol concentrations than those fed the $\mathrm{C}$ diet (Fig. 4(D)).

\section{Discussion}

$\mathrm{SP}$ is known to lead to significant reductions in serum and hepatic cholesterol concentrations in animal models and in humans. In the present study, as others have established in previous studies, we determined that one potential mechanism for the elimination of cholesterol by SP consumption is the elimination of $\mathrm{BA}^{(4,6,22)}$. There is evidence that multiple mechanisms are involved in the inhibition of CYP7A1 gene transcription via BA feedback. It is generally recognised that the FXR/SHP cascade mechanism mediates BA feedback inhibition of CYP7A1 in the liver ${ }^{(13,14)}$. SHP is a negative factor that interacts with many nuclear receptors, including LRH1. SHP inhibits the transactivating activity of LRH1, which binds to the CYP7A1 gene. We observed that rats fed the SP diet exhibited greater expression of $S H P$. However, the expression of $C Y P 7 A 1$ was higher only in the presence of $0 \cdot 2 \%$ cholesterol and SP. When CYP7A1 is deficient ${ }^{(23)}$, the secondary acid pathway of BA synthesis (CPY27A) compensates by generating and disposing of potentially hepatotoxic oxysterols derived from cholesterol ${ }^{(24,25)}$. We examined the expression of cholesterol $27 \alpha$-hydroxylase $(C Y P 27 A)$ and found no differences among the experimental groups. Furthermore, a SHP-independent mechanism has been described, involving FGF15 as a BA/FXR-induced intestinal hormone that inhibits CYP7A1 gene transcription in the liver ${ }^{(16)}$. FGF15 in the intestine is secreted into the circulatory system and then transported to the liver to activate the FGFR4 signalling pathway and inhibit CYP7A1 ${ }^{(26)}$. We detected high concentrations of FGF15 only in rats fed the SP diet. Interestingly, in the experimental groups fed diets containing $0 \cdot 2 \%$ cholesterol, FGF15 was not detected. However, when we measured FGFR4 concentrations in the liver of rats fed high-cholesterol diets, the concentration of the FGF15 receptor was found to be low. As a result, there was an increase in the concentration of faecal BA. These findings suggest that SP may regulate cholesterol homeostasis through a SHP-directed mechanism that decreases the expression of SHP and LRH1 and also through an indirect mechanism that decreases the secretion of FGF15 from the intestine and reduces the concentration of FGFR4 

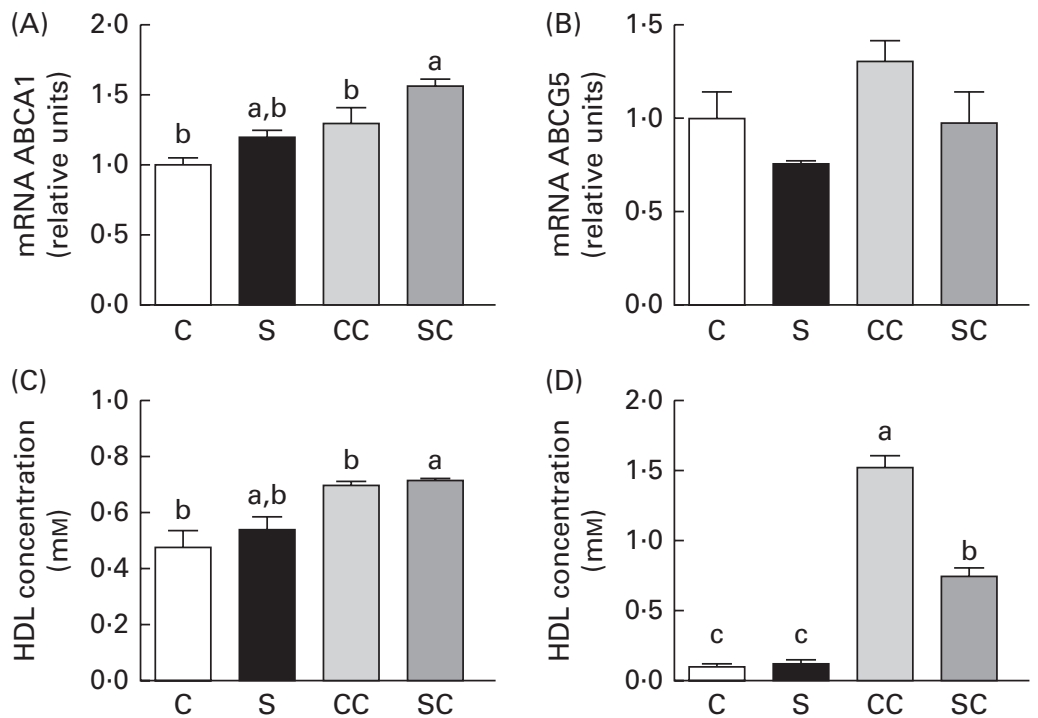

Fig. 3. Effects of the consumption of a soya protein diet with $0.2 \%$ cholesterol (SC) and without $0.2 \%$ cholesterol (S) for $9 \mathrm{~d}$ on the expression of (A) ATP-binding cassette transporter $\mathrm{A} 1$ (ABCA1) and (B) ATP-binding cassette, subfamily G, member 5 (ABCG5) in the liver of rats, (C) HDL concentrations in serum and (D) LDL concentrations in serum ( $n 12$ rats per group). Values are means, with their standard errors represented by vertical bars. ${ }^{\mathrm{a}, \mathrm{b}, \mathrm{c}}$ Mean values with unlike letters were significantly different $(P<0.05)$. C, $20 \%$ casein; CC, $20 \%$ casein plus $0.2 \%$ cholesterol.

in the liver. In both instances, an increase in the expression of $C Y P 7 A 1$, as well as in the synthesis and elimination of BA, led to a decrease in serum cholesterol concentrations.

RCT is generally described as the process by which cholesterol is removed from extrahepatic tissues and returned to the liver for conversion into BA and excretion into bile. Nonetheless, in the intestine, RCT plays a key role in the transport of excess cholesterol in the enterocytes into the intestinal lumen. Several ATP cassette-binding transporters, including ABCA1, ABCG5 and ABCG8, among others, are involved in RCT. It has been established that RCT is an important mechanism by which HDL protects against atherosclerosis ${ }^{(27)}$. Although ABCA1 facilitates the cellular efflux of cholesterol and phospholipids to lipid-poor apoAI or $\mathrm{HDL}^{(28)}$, we did not observe a significant change in HDL-cholesterol concentrations in rats fed the SP diet despite the modest increase in the expression of $A B C A 1$ mRNA. On the other hand, there was a small but significant increase in the expression of the $A B C G 5 / A B C G 8$ transporters involved in the secretion of sterols from the liver and the efflux of dietary sterols from the gut $^{(29)}$ to the liver; however, there was a significant increase in the mRNA abundance of both transporters in the intestine of rats fed the SP diet. Interestingly, this difference was magnified in rats fed the SC diet, suggesting that ABCG5 and ABCG8, as well as ABCA1, are regulated by the LXR/retinoid $\mathrm{X}$ receptor heterodimer because this nuclear receptor is activated by natural oxysterols ${ }^{(30)}$.

The increase in the excretion of faecal BA, due in part to the increase in BA synthesis promoted by an increase in the expression of $C Y P 7 A 1$ as well as an increase in the expression of the reverse cholesterol transporters in the intestine, could explain the well-established ability of SP to reduce serum cholesterol concentrations. In fact, our data revealed that rats fed a SP diet rich in cholesterol had significantly lower total and LDL-cholesterol concentrations in serum.
On the other hand, it is known that LRH1 interacts with LXR to regulate the expression of $S R E B P 1 C$, which is a master transcriptional factor that regulates lipogenesis. We observed that SP consumption reduced the expression of $L X R$ and SREBP1C, independently of the presence of cholesterol. These data may explain the reduction in hepatic TAG content observed in rats fed the SP diet. Furthermore, the reduction of SREBP1C expression in the liver of rats fed the SP diet independent of the presence or absence of cholesterol in the diet can be due to a decrease in insulin secretion, as the amino acid profile in the serum of rats fed SP diminishes the release of
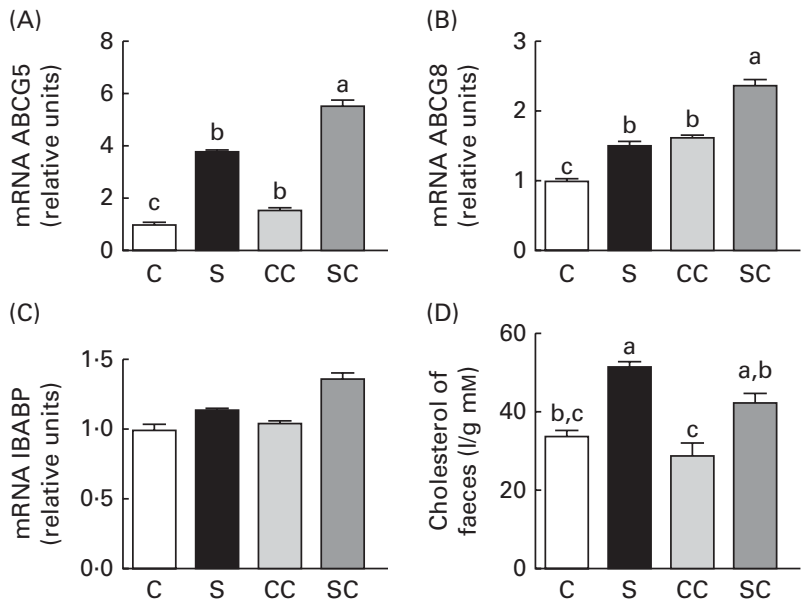

Fig. 4. Effects of the consumption of a soya protein diet with $0.2 \%$ cholesterol (SC) and without $0.2 \%$ cholesterol (S) for $9 \mathrm{~d}$ on the expression of (A) ATP-binding cassette, subfamily G, member 5 (ABCG5), (B) ATP-binding cassette, subfamily $\mathrm{G}$, member $8(A B C G 8)$ and $(C)$ ileal bile acid-binding protein in the ileum of rats and the concentrations of (D) total faecal cholesterol ( $n 12$ rats per group). Values are means, with their standard errors represented by vertical bars. ${ }^{a, b, c}$ Mean values with unlike letters were significantly different $(P<0.05)$. C, $20 \%$ casein; CC, $20 \%$ casein plus $0.2 \%$ cholesterol. 

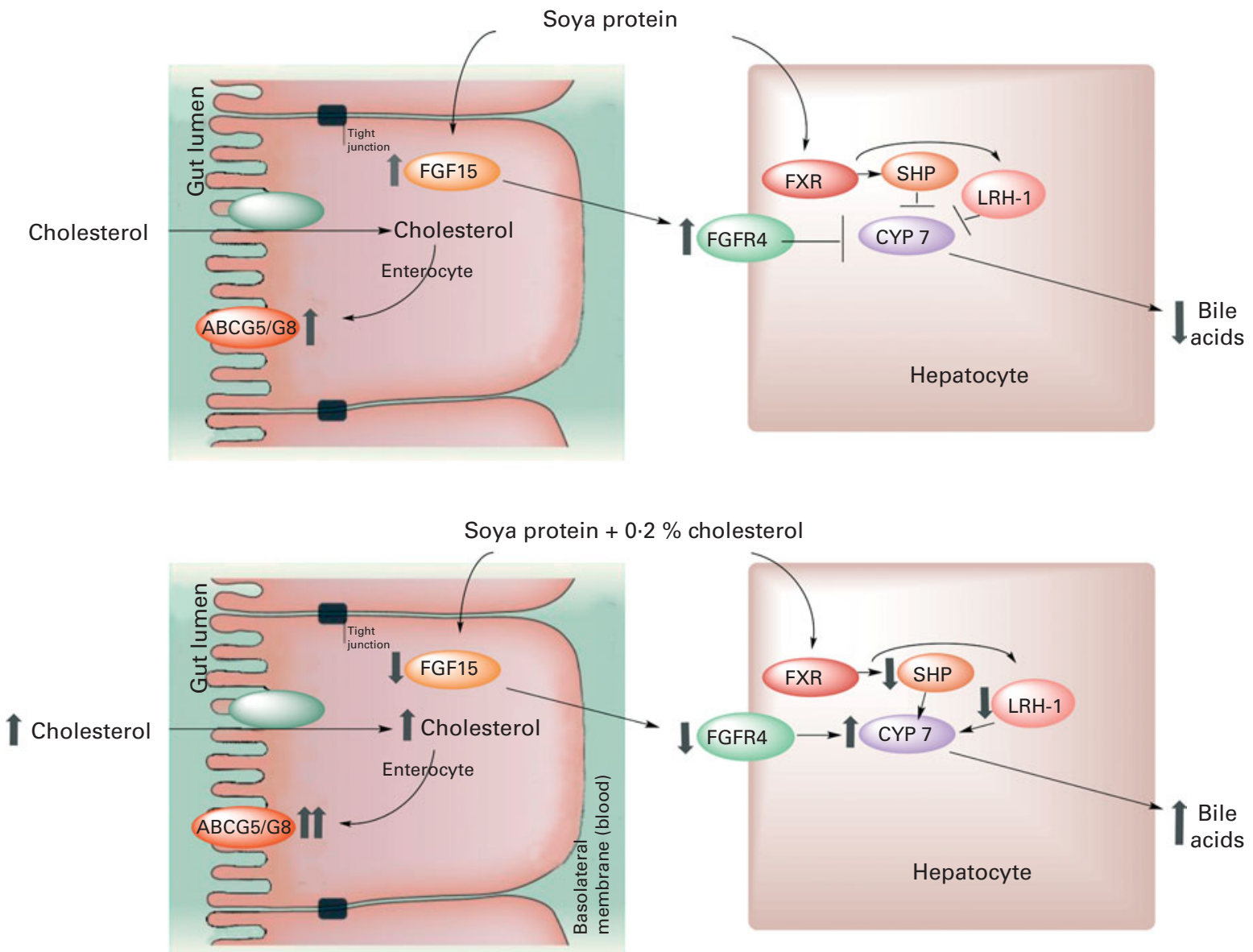

Fig. 5. Proposed model for the mechanism by which soya protein regulates reverse cholesterol transport and bile acid synthesis via fibroblast growth factor 15 (FGF15) and/or SHP after the consumption of soya protein (A) and soya protein plus $0.2 \%$ cholesterol (B) compared with that in the control groups fed casein or casein plus $0.2 \%$ cholesterol, respectively. In the absence of cholesterol in the diet, soya protein stimulates the expression of intestinal FGF15 as well as its hepatic receptor fibroblast growth factor receptor 4 (FGFR4). In addition, soya protein also induces the expression of SHP and liver receptor homolog 1 (LRH1). Both mechanisms decrease the expression of $7 \alpha$-hydroxylase (CYP7A1) and hence the excretion of bile acids. The addition of cholesterol to a soya protein diet reverses this process by increasing the excretion of faecal bile acids and cholesterol via the up-regulation of the expression of intestinal reverse cholesterol transporters ATP-binding cassette, subfamily G, member 5/8 (ABCG5/ABCG8). FXR, farnesoid X receptor.

pancreatic insulin as has been reported previously, a hormone involved in the up-regulation of SREBP1C expression ${ }^{(31)}$. Interestingly, the addition of cholesterol to the diets increased the concentrations of hepatic TAG. It has been demonstrated that cholesterol can increase the expression of SREBP1C via $L X R^{(32)}$, and we observed a significant increase in SREBP $1 c$ expression in rats fed the CC diet, although rats fed the SC diet exhibited a modest increase.

In conclusion, there are two possible mechanisms by which SP regulates cholesterol homeostasis: (1) by increasing BA synthesis via a direct SHP mechanism, by decreasing the expression of SHP and LRH1 and consequently increasing the expression of CYP7A1, and by decreasing the secretion of FGF15 and the hepatic receptor FGFR4, which favours an increase in CYP7A1 expression, or (2) by increasing the activity of LXR, leading to lower serum LDL concentrations by increasing the expression of $A B C A 1$ in the liver while promoting RCT in the intestine by increasing the expression of the $A B C G 5$ / $A B C G 8$ transporter (Fig. 5). Further studies are needed to understand how isoflavones are involved in these mechanisms.

\section{Acknowledgements}

The present study was supported by the CONACYT grant 154939 to A. R. T. The funder contributed to the study design and conduct and manuscript preparation.

The authors' contributions are as follows: G. L. A.-M. was responsible for the experimental design, analysed the data and wrote the manuscript; N. T. was responsible for the experimental design and data analysis; O. G. wrote the manuscript and analysed the data; B. P.-G. wrote the manuscript and analysed the data; I. M.-V. analysed the data; A. R. T. contributed to the experimental design and discussion and wrote the manuscript.

\section{References}

1. Anderson JW, Johnstone BM \& Cook-Newell ME (1995) Meta-analysis of the effects of soy protein intake on serum lipids. N Engl J Med 333, 276-282.

2. Reynolds K, Chin A, Lees KA, et al. (2006) A meta-analysis of the effect of soy protein supplementation on serum lipids Am J Cardiol 98, 633-640. 
3. Zhan S \& Ho SC (2005) Meta-analysis of the effects of soy protein containing isoflavones on the lipid profile. $\mathrm{Am} \mathrm{J}$ Clin Nutr 81, 397-408.

4. Potter SM (1995) Overview of proposed mechanisms for the hypocholesterolemic effect of soy. J Nutr 125, 606S-611S.

5. Torres N, Torre-Villalvazo I \& Tovar AR (2006) Regulation of lipid metabolism by soy protein and its implication in diseases mediated by lipid disorders. J Nutr Biochem 17, 365-373.

6. Lin Y, Meijer GW, Vermeer MA, et al. (2004) Soy protein enhances the cholesterol-lowering effect of plant sterol esters in cholesterol-fed hamsters. J Nutr 134, 143-148.

7. Tanaka K, Aso B \& Sugano M (1984) Biliary steroid excretion in rats fed soybean protein and casein or their amino acid mixtures. J Nutr 114, 26-32.

8. Chawla A, Saez E \& Evans RM (2000) "Don't know much bile-ology". Cell 103, 1-4.

9. Russell DW (2003) The enzymes, regulation, and genetics of bile acid synthesis. Annu Rev Biochem 72, 137-174.

10. Repa JJ, Lund EG, Horton JD, et al. (2000) Disruption of the sterol 27-hydroxylase gene in mice results in hepatomegaly and hypertriglyceridemia. Reversal by cholic acid feeding. J Biol Chem 275, 39685-39692.

11. Peet DJ, Turley SD, Ma W, et al. (1998) Cholesterol and bile acid metabolism are impaired in mice lacking the nuclear oxysterol receptor LXR alpha. Cell 93, 693-704.

12. Sinal CJ, Tohkin M, Miyata M, et al. (2000) Targeted disruption of the nuclear receptor FXR/BAR impairs bile acid and lipid homeostasis. Cell 102, 731-744.

13. Lu TT, Makishima M, Repa JJ, et al. (2000) Molecular basis for feedback regulation of bile acid synthesis by nuclear receptors. Mol cell 6, 507-515.

14. Goodwin B, Jones SA, Price RR, et al. (2000) A regulatory cascade of the nuclear receptors FXR, SHP-1, and LRH-1 represses bile acid biosynthesis. Mol Cell 6, 517-526.

15. Xu G, Salen G, Shefer S, et al. (1999) Increasing dietary cholesterol induces different regulation of classic and alternative bile acid synthesis. J Clin Invest 103, 89-95.

16. Inagaki $\mathrm{T}$, Choi $\mathrm{M}$, Moschetta A, et al. (2005) Fibroblast growth factor 15 functions as an enterohepatic signal to regulate bile acid homeostasis. Cell Metab 2, 217-225.

17. Nakahara M, Furuya N, Takagaki K, et al. (2005) Ileal bile acid-binding protein, functionally associated with the farnesoid $\mathrm{X}$ receptor or the ileal bile acid transporter, regulates bile acid activity in the small intestine. $J$ Biol Chem $\mathbf{2 8 0}$, 42283-42289.

18. Kamisako T \& Ogawa H (2003) Regulation of biliary cholesterol secretion is associated with $a b c g 5$ and $\operatorname{abcg} 8$ expressions in the rats: effects of diosgenin and ethinyl estradiol. Hepatol Res 26, 348-352.

19. Folch J, Lees M \& Sloane Stanley GH (1957) A simple method for the isolation and purification of total lipides from animal tissues. J Biol Chem 226, 497-509.

20. Turley SD \& Dietschy JM (1978) Re-evaluation of the 3 alphahydroxysteroid dehydrogenase assay for total bile acids in bile. J Lipid Res 19, 924-928.

21. Chomczynski P \& Sacchi N (1987) Single-step method of RNA isolation by acid guanidinium thiocyanate-phenolchloroform extraction. Anal Biochem 162, 156-159.

22. Huff MW \& Carroll KK (1980) Effects of dietary protein on turnover, oxidation, and absorption of cholesterol, and on steroid excretion in rabbits. J Lipid Res 21, 546-548.

23. Schwarz M, Lund EG, Setchell KD, et al. (1996) Disruption of cholesterol 7alpha-hydroxylase gene in mice. II. Bile acid deficiency is overcome by induction of oxysterol 7alphahydroxylase. J Biol Chem 271, 18024-18031.

24. Bjorkhem I (1992) Mechanism of degradation of the steroid side chain in the formation of bile acids. J Lipid Res 33, 455-471.

25. Schwarz M, Lund EG, Lathe R, et al. (1997) Identification and characterization of a mouse oxysterol 7alpha-hydroxylase cDNA. J Biol Chem 272, 23995-24001.

26. Yu C, Wang F, Kan M, et al. (2000) Elevated cholesterol metabolism and bile acid synthesis in mice lacking membrane tyrosine kinase receptor FGFR4. J Biol Chem 275 , 15482-15489

27. Fielding CJ \& Fielding PE (1997) Intracellular cholesterol transport. J Lipid Res 38, 1503-1521.

28. Oram JF \& Vaughan AM (2000) ABCA1-mediated transport of cellular cholesterol and phospholipids to HDL apolipoproteins. Curr Opin Lipidol 11, 253-260.

29. Berge KE, Tian H, Graf GA, et al. (2000) Accumulation of dietary cholesterol in sitosterolemia caused by mutations in adjacent ABC transporters. Science 290, 1771-1775.

30. Lee MH, Lu K, Hazard S, et al. (2001) Identification of a gene, $A B C G 5$, important in the regulation of dietary cholesterol absorption. Nat Genet 27, 79-83.

31. Noriega-Lopez L, Tovar AR, Gonzalez-Granillo $\mathrm{M}$, et al. (2007) Pancreatic insulin secretion in rats fed a soy protein high fat diet depends on the interaction between the amino acid pattern and isoflavones. I Biol Chem 282, 20657-20666

32. Lin J, Yang R, Tarr PT, et al. (2005) Hyperlipidemic effects of dietary saturated fats mediated through PGC-1beta coactivation of SREBP. Cell 120, 261-273. 